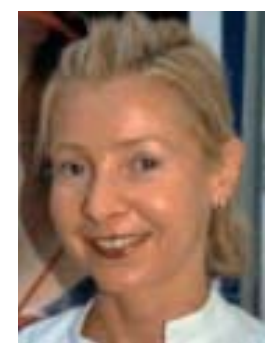

Verena Henkel

\section{Früherkennung depressiver Störungen in der
Primärversorgung}

Verena Henkel, Roland Mergl, Michael Schütze, Antje-Kathrin Allgaier, Ralf Kohnen, Ulrich Hegerl

Psychiatrische Klinik und Poliklinik der Ludwig-Maximilians-Universität München

psychoneuro 2003; 29 (1): 35-39
D epressive Störungen stellen in der Hausarztpraxis die am häufigsten gesehene psychiatrische Erkrankung dar. Hier werden die Weichen für die Diagnostik, die Behandlung und somit auch für den weiteren Verlauf der Erkrankung gestellt. Gerade in der hausärztlichen Praxis bereitet aber die diagnostische Zuordnung Schwierigkeiten $(9,13)$. Die meisten depressiven Patienten thematisieren während des Gesprächs mit dem Arzt überwiegend körperliche Beschwerden. Insbesondere ältere Patienten bagatellisieren häufig depressive Symptome und sprechen diese eher ungern an. Ein Grund dafür liegt darin, dass depressive Patienten selbst ihren Zustand sehr oft als persönliches, schuldhaftes Versagen und eher nicht als Erkrankung erleben. Zahlreiche Studien belegen, dass bei etwa der Hälfte der Patienten die depressive Störung nicht erkannt wird (9). Einer Verbesserung der Diagnostik depressiver Störungen in der Primärversorgung kommt daher besondere Bedeutung zu.

In der vorliegenden Studie wurde untersucht, inwieweit die Diagnosestellung durch den Einsatz eines Fragebogens optimiert werden kann (6). In diesem Zusammenhang wurden drei psychometrische In-

Etwa 20\% aller Patienten in der Primärversorgung leiden unter einer depressiven Störung. Bis zu 50\% dieser Erkrankungen bleiben unentdeckt. Unser Ziel war es, eine praktikable Methode zu finden, mit der die Früherkennung von Depressionen in hausärztlichen Praxen erleichtert wird. Hierfür wurden in einer Kohortenstudie drei Screeninginstrumente hinsichtlich ihrer Validität miteinander verglichen. Der kürzeste Fragebogen (WHO-5 Well-Being Index) zeigte dabei die höchste Sensitivität (94\%, Spezifität: 65\%). Das Ergebnis unterstützt die Empfehlung der Weltgesundheitsorganisation, möglichst alle Patienten in der Primärversorgung mit diesem Fragebogen in regelmäßigen Abständen zu untersuchen. Ein solches Routinescreening würde zusätzlich ein verstärktes Bewusstsein für das mögliche Vorliegen einer Depression in der Primärversorgung schaffen.

strumente hinsichtlich ihrer Validität (Sensitivität, Spezifität) verglichen. Es wurde also untersucht, wie gut sich mit diesen Fragebögen die Erkrankung Depression erkennen lässt. Aus der Vielzahl zur Verfügung stehender Screeninginstrumente wurden drei kurze Selbstbeurteilungsfragebögen ausgewählt:

- Ein krankheitsspezifischer Fragebogen: das Depressionsmodul des neun Items umfassenden „Brief Patient Health Questionnaire“ („B-PHQ“) (10)

- Ein sehr allgemeiner Fragebogen: der aus zwölf Items bestehende „General Health Questionnaire“ („GHQ-12“) (4)

- Der nur fünf Fragen umfassende "WHO-5 Well-Being Index“ („WHO-5“) (14): der im Hinblick auf die Krankheitsspezifität etwa zwischen den beiden anderen Fragebögen anzusiedeln ist.

\section{Methodik Patienten}

487 Patienten aus 18 Hausarztpraxen in Nürnberg willigten nach ausführlicher Aufklärung in die Teilnahme an der Untersuchung ein. Die Altersgrenzen lagen bei 18 bzw. 90 Jahren, ein positives Votum der Ethikkommission lag vor. Soweit durchführbar, wurden alle Patienten, die sich an einem bestimmten Stichtag im Wartezimmer ihres Hausarztes vorstellten, untersucht (Kohortenstudiendesign). Die Patienten wurden gebeten, sämtliche Items der drei Fragebögen (WHO-5, B-PHQ, GHQ-12) vor der ärztlichen Konsultation auszufüllen. Kurz nach dem Praxisbesuch wurden die Pa- 
tienten von speziell trainierten ärztlichen und psychologischen Mitarbeitern telefonisch kontaktiert. Diesen Mitarbeitern waren die Ergebnisse der Fragebögen nicht bekannt. Sie befragten die Patienten, indem sie ein standardisiertes psychiatrisches Interview („Composite International Diagnostic Interview“ („CIDI“) (14)) durchführten. 17 Patienten, die das Zeitkriterium für das ihr Einverständnis zurückzogen, wurden aus der Studie ausgeschlossen. 22 Patienten hatten die Screeninginstrumente nur unvollständig ausgefüllt. Die Validitätsanalyse der Screeninginstrumente wurde daher nur auf der Basis von 448 kompletten Datensätzen durchgeführt (Tab. $1)$. CIDI-Interview nicht erfüllten oder

Psychometrische Instrumente: Fragebögen und Interview

Composite International Diagnostic Interview (CIDI): Das Interview wurde auf Grund seiner guten Reliabilität und Validität als Referenzstandard ausgewählt (1), d.h. die Ergebnisse der Fragebögen wurden jeweils mit dem Ergebnis des Interviews verglichen. Wir verwendeten eine Softwareversion (DIA-X) (12), basierend auf der CIDI-Version 1.1 (14).

Der B-PHQ wurde Ende der 90er Jahre von Spitzer entwickelt (10). Wir begrenzten die Untersuchung auf das Depressionsmodul, welches alle neun DSM-IV-Kriterien für eine majore depressive Störung abfragt; die Auswertung erfolgte nach den Vorgaben von Spitzer (10). Wir benutzten die Übersetzung von Löwe

\section{Abb. 1 Vergleich dreier Screeninginstrumente}

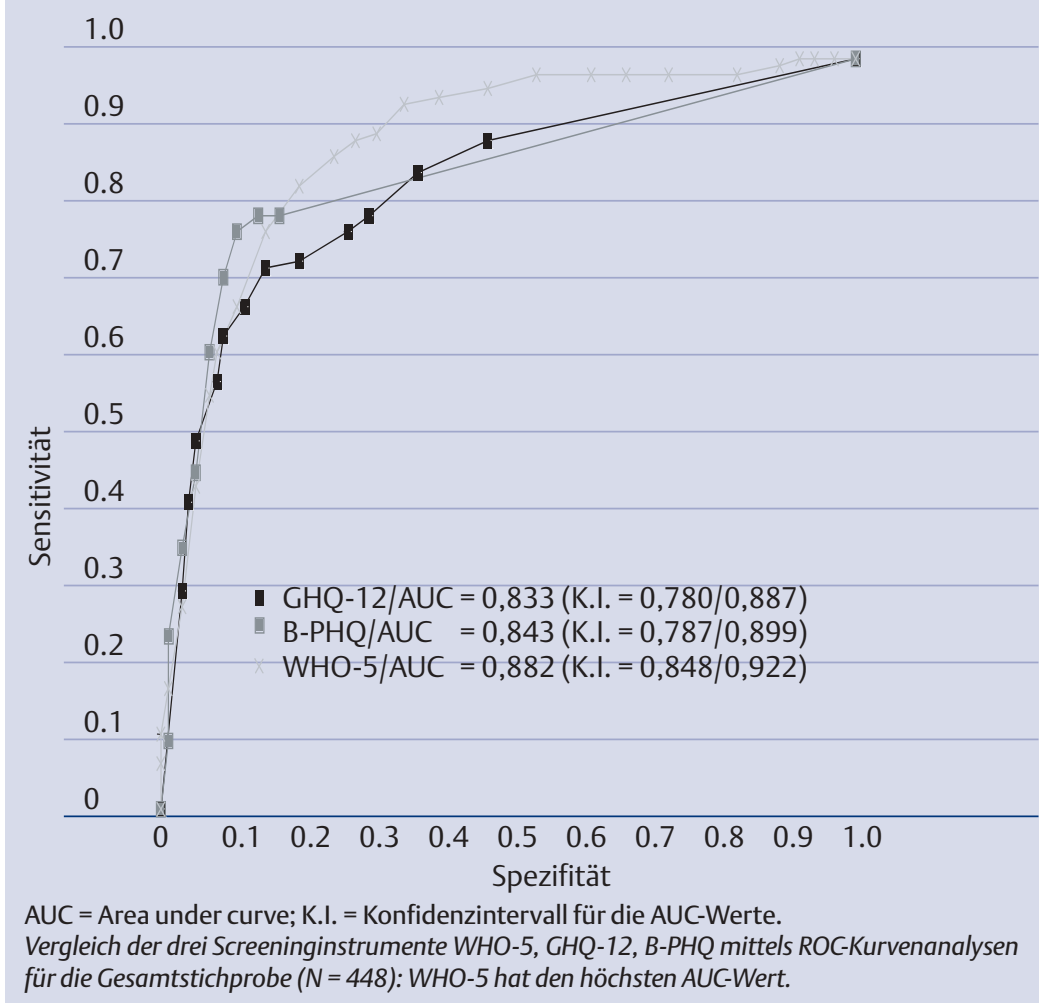

\section{Tab.1 Beschreibung der Gesamtstichprobe*}

\begin{tabular}{|c|c|c|}
\hline & $\begin{array}{l}\text { Depression nach CIDI } \\
\text { (gemäß ICD-10-Kriterien) }\end{array}$ & $\begin{array}{l}\text { Keine Depression nach CIDI } \\
\text { (gemäß ICD-10-Kriterien) }\end{array}$ \\
\hline $\mathrm{N}(\%)$ & $82(18,3 \%)$ & $366(81,7 \%)$ \\
\hline Alter in Jahren $(\mathrm{M} \pm \mathrm{s})$ & $47,2( \pm 15,0)$ & $54,1( \pm 16,7)$ \\
\hline Geschlecht & $57 \mathrm{~F} / 25 \mathrm{M}$ & $226 F / 140 M$ \\
\hline
\end{tabular}

und Mitarbeitern (8). Der WHO-5Index zum Wohlbefinden wurde in den 90er Jahren von Bech vorgestellt, mit fünf kurzen, positiv formulierten, nicht depressionsspezifischen Items, die das Ausmaß des Wohlfühlens (Well-Being) messen; die Auswertung erfolgte nach den Vorgaben der WHO (15). Der „General Health Questionnaire“ (GHQ-12) wurde bereits in den 70er Jahren von Goldberg entwickelt (4), als Screeninginstrument für nicht-psychotische psychische Erkrankungen.

\section{Statistische Analyse}

Für jedes Screeninginstrument wurden die in der Literatur empfohlenen Standard-Grenzwerte („Cutoff“-Werte) gewählt. Auf der Grundlage von Vierfeldertafeln wurden Sensitivität und Spezifität sowie positiver und negativer prädiktiver Wert errechnet, um die Fähigkeit der Screeninginstrumente zu prüfen, eine depressive Störung korrekt zu klassifizieren.

ROC-Kurven wurden konstruiert, um die Screeninginstrumente hinsichtlich ihrer diagnostischen Validität unabhängig von a priori definierten Grenzwerten vergleichen zu können. ROC-Kurven und die Flächen unter den Kurven (AUCWerte) wurden mit Hilfe der Statistiksoftware SPSS für Windows (Version 10.0) berechnet.

Die Fähigkeit eines Screeninginstrumentes, eine depressive Störung zu erkennen, kann durch verschiedene Faktoren beeinflusst werden. Von daher kann nicht einfach davon ausgegangen werden, dass Sensitivitäten und Spezifitäten in verschiedenen Untergruppen immer gleich sind. Daher führten wir auch Analysen in Subgruppen durch und konzentrierten uns dabei auf die zwei demographischen Variablen Geschlecht und Alter, da Hinweise bestehen, dass sich Depressionen bei Männern und Frauen (2, 3) sowie bei älteren und jüngeren Patienten klinisch unterscheiden (7).

\section{Ergebnisse}

Tabelle 2 fasst die Ergebnisse zur diagnostischen Validität der drei Screeninginstrumente B-PHQ, GHQ12 und WHO-5 - ausgehend von be- 
reits etablierten Schwellenwerten („Cut-off“-Werten) - zusammen. Der WHO-5 ist den beiden anderen Fragebögen in dem für den Screeningprozess wichtigsten Parameter Sensitivität (Anteil der richtig erkannten Depressionen) eindeutig überlegen. Auf der anderen Seite zeigen die niedrigeren Werte für die Spezifität (Anteil der richtig als gesund erkannten Fälle), dass nicht wenigen Patienten durch den WHO-5 fälschlicherweise eine depressive Störung zugeordnet wurde. Dieses Problem besteht auch beim GHQ-12, aber nicht so sehr beim B-PHQ. Die Spezifität und der positive prädiktive Wert sind beim B-PHQ höher als bei den beiden anderen Instrumenten.

Alle Messwerte in Tabelle 2 hängen entscheidend von der Wahl des jeweiligen diagnostischen Schwellenwertes ab. Im Folgenden wurde die ROC-Analyse zum Vergleich der drei Screeninginstrumente eingesetzt, da ROC-Kurven unabhängig von der Festlegung eines einzelnen diagnostischen Schwellenwertes sind.

Abbildung 1 zeigt die ROC-Kurven für jedes einzelne Screeninginstrument bezogen auf die gesamte Stichprobe. Die Fläche unter jeder einzelnen ROC-Kurve dient jeweils als Maß für die Güte des Instruments (AUC: Area under the curve). Bei einer zufälligen Übereinstimmung der CIDI-Diagnose mit der Diagnose der Screeninginstrumente würde ein AUC-Wert bei 0,5 liegen. In unserer Untersuchung aber liegen sämtliche AUC-Werte über 0,8. Dies zeigt eine ausreichende Validität aller drei Screeningmethoden an, mit dem besten Ergebnis für den WHO-5 $(0,88)$.

In unserer ROC-Analyse der Untergruppen konzentrierten wir uns auf die beiden demographischen Variablen Geschlecht und Alter. Der WHO-5 zeigte hier besonders gute Ergebnisse in der Untergruppe der weiblichen und der älteren Patienten (AUC-Wert Frauen: 0,90; AUCWert ältere Patienten: 0,88).

Zusammenfassend zeigt sich der WHO-5 als sehr gutes Screeninginstrument.

\section{Diskussion}

Anwendung des WHO-5-Frage-

bogens in der Primärversorgung

Die hohe Sensitivität und der günstige AUC-Wert des WHO-5, zusammen mit seiner Kürze, seiner positiven Formulierung und seiner einfachen Handhabung, ergeben, dass dieser Test ein nützliches Screeninginstrument zur Identifizierung depressiver Patienten in der Primärversorgung ist (Abb. 2).

Die Ergebnisse dieser Studie unterstützen damit die Empfehlung der WHO, jeden Patienten in der Primärversorgung in regelmäßigen Zeitintervallen zu bitten, an einer Untersuchung mit dem WHO-5-Fragebogen teilzunehmen. Die Patienten können die fünf Fragen schon im Wartezimmer ausfüllen (als ersten Schritt im Screeningprozess). Der WHO-5 könnte so analog zu bereits bestehenden Screeningverfahren, wie z.B. bei Diabetes mellitus oder Hypertonie, angewendet und leicht ausgewertet werden (z.B. auch durch Praxisassistenten). Die bezüglich einer depressiven Störung positiv gescreenten Patienten sollten in einem zweiten Untersuchungsgang - dem sich nun anschließenden diagnostischen Interview - weiter abgeklärt werden (Abb. 3). Im ärztlichen Gespräch kann jetzt auf das Ergebnis des WHO-5-Fragebogens Bezug genommen werden und so der Einstieg in die Thematik erleichtert werden. Der Arzt sollte rein körperliche Ursachen der Depression oder normale Konflikt-, Verlust- oder Trauerreaktionen ausschließen oder aber die Diagnose einer Depression bestätigen. Auch das Vorliegen manischer

\section{Tab.2 Vergleich dreier Screeningfragebögen hinsichtlich ihrer diagnostischen Gütekriterien}

\begin{tabular}{|c|c|c|c|c|}
\hline Fragebogen* & Sensitivität & Spezifität & PPW $^{* *}$ & $\mathbf{N P W}^{* * *}$ \\
\hline B-PHQ ( $\geq 2$, inkl. 1a or $1 b)$ & 0,79 & 0,86 & 0,55 & 0,95 \\
\hline GHQ-12( $(\geq 2)$ & 0,85 & 0,63 & 0,34 & 0,95 \\
\hline WHO-5 ( $\leq 13)$ & 0,94 & 0,65 & 0,37 & 0,98 \\
\hline $\begin{array}{l}{ }^{*} \text { Auswertung wie in der Fachli } \\
{ }^{* *} \mathrm{PPW}=\text { Positiver prädiktiver } \\
{ }^{* * *} \mathrm{NPW}=\text { Negativer prädikti }\end{array}$ & $\begin{array}{l}\text { tur empfohlen. } \\
\text { t } \\
\text { Vert }\end{array}$ & & & \\
\hline
\end{tabular}

\section{Abb. 2 WHO-5 Index zum Wohlbefinden}

\begin{tabular}{l|l|l|l|l|l}
$\begin{array}{l}\text { In den letzten } \\
\text { beiden Wochen }\end{array}$ & Die ganze Zeit & Meistens & $\begin{array}{l}\text { Über die } \\
\text { Hälfte der Zeit }\end{array}$ & $\begin{array}{l}\text { Weniger als die } \\
\text { Hälfte der Zeit }\end{array}$ & $\begin{array}{l}\text { Ab und zu } \\
\text { 1. Ich war froh und } \\
\text { guter Laune }\end{array}$ \\
$\begin{array}{l}\text { 2. Ich habe mich ruhig und } \\
\text { entspannt gefühlt }\end{array}$ & & & & \\
\hline $\begin{array}{l}\text { 3. Ich habe mich aktiv und } \\
\text { voller Energie gefühlt }\end{array}$ & & & & & \\
\hline $\begin{array}{l}\text { 4. Beim Aufwachen habe } \\
\text { ich mich frisch und } \\
\text { ausgeruht gefühlt }\end{array}$ & & & & & \\
\hline $\begin{array}{l}\text { 5. Mein Alltag war voller } \\
\text { Dinge, die mich } \\
\text { interessieren }\end{array}$ & & & & & \\
\hline
\end{tabular}


Episoden in der Vorgeschichte sollte geprüft werden, bevor eine Behandlungsentscheidung getroffen wird. In diesem zweiten Schritt könnte der spezifische B-PHQ als kurze Checkliste von den Ärzten genutzt werden und zusätzlich Unterstützung bieten.

\section{Depression bei älteren \\ Patienten}

Für den WHO-5 sprechen auch die hinsichtlich der Sensitivität guten Testwerte bei älteren Patienten. In dieser Gruppe sind die diagnostischen und therapeutischen Defizite besonders gravierend, obwohl auch für diese Patientengruppe effektive Therapien zur Verfügung stehen (5).

\section{Kostenaspekte}

Das oben beschriebene einfache und kostengünstige Routinescreening-Programm sollte von Fortbildungen begleitet sein, um Ärzte in der Primärversorgung mit diagnostischen psychiatrischen Interviews vertraut zu machen und um sie bei der Behandlung depressiver Patienten zu unterstützen. Auch wäre es sinnvoll, Therapierichtlinien zu etablieren, die die Besonderheiten der Primärversorgung berücksichtigen. Diese Anstrengungen würden sich bezahlt machen, da Kosten-Nutzen-Analysen des Depressionsscreenings in der Primärversorgung gezeigt haben, dass die daraus resultierende Verbesserung der Versorgungsqualität bei depressiven Patienten erheblich zur Kostensenkung in diesem Bereich des Gesundheitswesens beitragen kann (11).


Prävalenzrate von Depressionen in der Primärversorgung

Ein auffälliges Ergebnis unserer Studie ist die hohe Punktprävalenzrate depressiver Störungen (18,3\%) in unserer Stichprobe. Diese Rate umfasst das gesamte Spektrum depressiver Störungen, einschließlich solcher Erkrankungen wie dysthyme Störung, kurze rezidivierende depressive Störung, substanzinduzierte depressive Störung usw. Die in epidemiologischen Studien angegebenen Prävalenzraten von Depressionen in der Primärversorgung variieren erheblich. Eine ähnliche Prävalenzrate (16\%) für die Diagnose irgendeiner Art von affektiver Störung in der Primärversorgung wurde in der „PHQ Primary Care Study" von Spitzer et al. (10) gefunden. Trotzdem ist die Punktprävalenz in unserer Studie höher als in epidemiologischen Untersuchungen im Bereich der Primärversorgung, die in anderen deutschen Städten durchgeführt wurden (mit Prävalenzraten zwischen 5 und 10\%). Die eher hohe Rate in unserer Studie könnte auch Folge dessen sein, dass vor allem Praxen mit Interesse an depressiven Störungen und einem entsprechenden Klientel sich zu der Teilnahme an unserer Studie bereit erklärten. Selektionseffekte bei der Auswahl der Patienten in den Arztpraxen können ebenfalls nicht vollständig ausgeschlossen werden. Stark variierende Prävalenzzahlen vermindern jedoch nicht die Tragweite unserer Ergebnisse, da die Hauptkriterien für den Vergleich von Screeninginstrumenten, nämlich Sensitivität und Spezifität, von Prävalenzraten unabhängig sind.

\section{Zukunftsperspektive}

Wir hoffen, dass die Ergebnisse der vorliegenden Studie, welche den WHO-5 als nützliches Instrument für das Screening depressiver Störungen in der Primärversorgung favorisiert, zu weiteren Anstrengungen in der nationalen und internationalen Forschung auf diesem wichtigen Gebiet des Gesundheitswesens führen werden.

\section{Danksagung}

Dieses Projekt wird vom Bundesministerium für Bildung und Forschung (BMBF) im Rahmen des Förderschwerpunktes „Kompetenznetze in der Medizin“ gefördert.

About $20 \%$ of all patients in primary care suffer from any depressive disorder. Up to $50 \%$ of these diseases remain undetected. Our research aimed at improving early recognition of depression in primary care settings. In this context, we compared three different screening tests in order to evaluate which would be the most appropriate one for the use in primary care settings. In a cohort study, the validity of each test was computed. The shortest screening instrument (WHO-5 Well-Being Index) had the highest sensitivity (94\%, specificity 65\%). The results support the recommendation of the World Health Organisation to screen all patients in primary care using the WHO-5 Well-Being Index questionnaire in a routine two-stage screening process. Thus, primary care physicians would be even more aware that their patients might suffer from a depressive disorder.

\section{Abb. 3 Zweistufiger Screeningprozess zur Identifikation depressiver Patienten in der ärztlichen Primärversorgung}
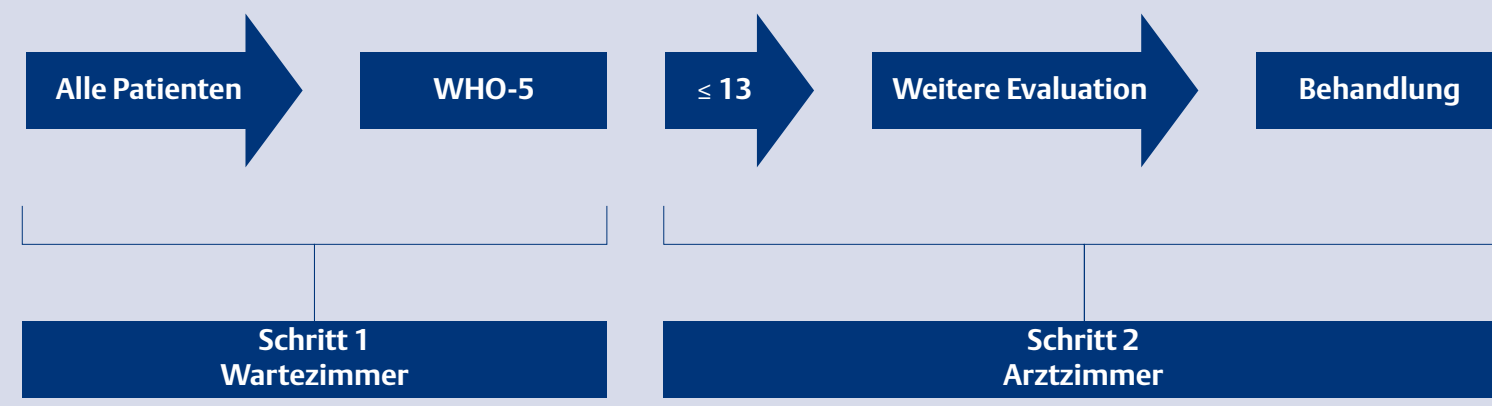
Key Words

Primary care - depressive disorder routine screening process - WHO-5

Well-Being Index questionnaire

\section{Literatur}

1. Andrews G, Peters L. The psychometric properties of the Composite International Diagnostic Interview. Soc Psychiatry Psychiatr Epidemiol 1998; 33: 80-88

2. Bech P. Male depression: stress and aggression as pathways to major depression. In: Dawson A, Tylee A (Hrsg.). Depression: Social and economic timebomb. London, British Medical Journal Books, 2001: 63-66

3. Dawson A, Tylee A. Depression: Social and economic timebomb. London, British Medical Journal Books, 2001.

4. Goldberg D. Manual of the General Health Questionnaire. Windsor, NFER Publishing Company, 1978.

5. Henkel V, Hegerl U. Altersdepression eine unterschätzte Gefahr. Geburtshilfe und Frauenheilkunde 2000; 60: 185-188

6. Henkel V, Mergl R et al. Identifying depression in primary care: A comparison of different methods in a prospective cohort study. BM] 2002 (im Druck)

7. Heun R, Burkart M et al. Internal and external validity of the WHO Well-Being Scale in the elderly general population. Acta Psychiatr Scand 1999; 99: 171-178

8. Löwe B, Spitzer RL et al. Gesundheitsfragebogen für Patienten (PHQ-D). Manua und Testunterlagen. [PRIME MD Patient Health Questionnaire (PHQ) - German version. Manual and materials]. Karlsruhe, Pfizer, 2001.

9. Paykel ES, Tylee A et al. The Defeat Depression Campaign: psychiatry in the public arena. Am J Psychiatry 1997; 154(6Suppl): 59-65

10. Spitzer RL, Kroenke K et al. Validation and utility of a self-report version of PRIMEMD: the PHQ primary care study. Primary Care Evaluation of Mental Disorders. Patient Health Questionnaire. JAMA 1999; 282: 1737-1744

11. Valenstein M, Vijan S et al. The cost-utility of screening for depression in primary care. Ann Intern Med 2001; 134 (5): 345-360

12. Wittchen $\mathrm{HU}$, Pfister $\mathrm{H}$. Instruktionsmanual zur Durchführung von DIA-X Interviews. Frankfurt am Main, Swets Test Services.

13. Wittchen HU, Winter $S$ et al. Häufigkeit und Erkennensrate von Depressionen in de hausärztlichen Praxis. MMW-Fortschritte der Medizin 2000; 118, Sonderheft I: 22-30 14. World Health Organization Info Package. Composite International Diagnostic Interview, Version 1.1. Genf, World Health Organization.

15. World Health Organization Info Package. Mastering depression in primary care. Frederiksborg: World Health Organization, Regional Office for Europe, Psychiatric Research Unit, 1998.

\section{Korrespondenzadresse:}

Dr. med. Verena Henkel

Psychiatrische Klinik und Poliklinik der Ludwig-Maximilians-Universität München

Nußbaumstr. 7

80336 München

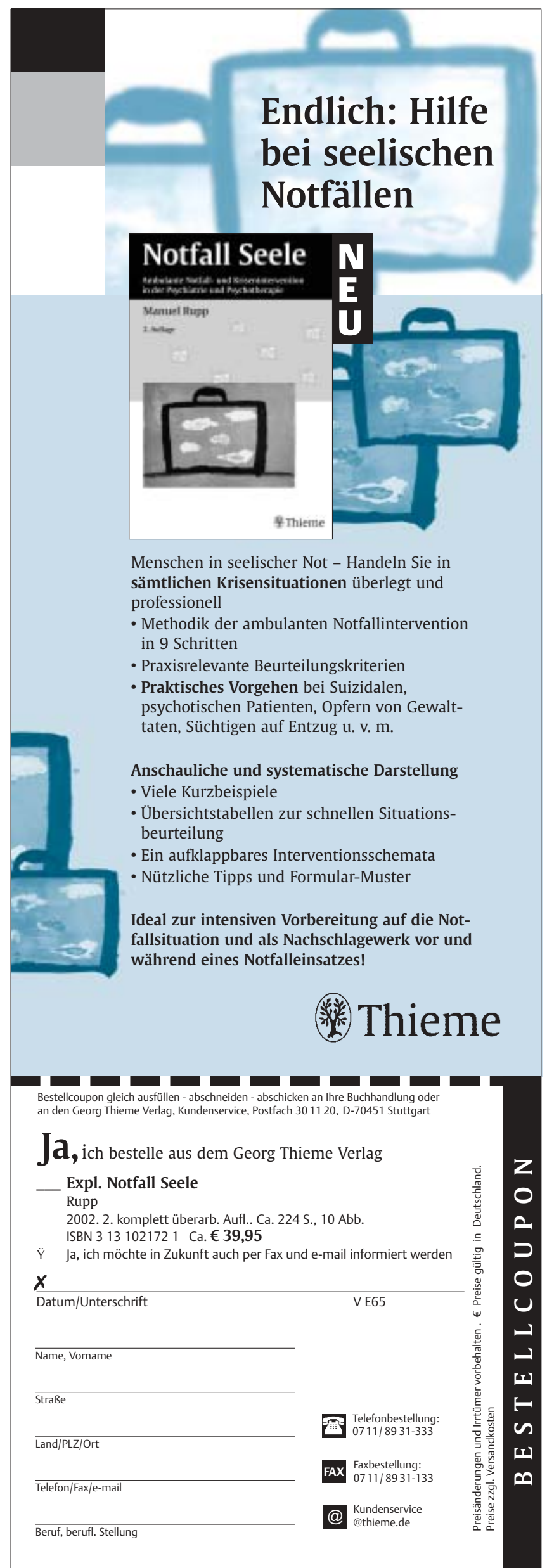

